\title{
Clocking of stators in one and half stage of axial steam turbine
}

\author{
Martin Němec ${ }^{1, *}$, Tomáš Jelínek ${ }^{1}$, and Petr Milčák ${ }^{2}$ \\ ${ }^{1}$ VZLÚ, Beranových 130, Prague 9, Czech Republic \\ ${ }^{2}$ Doosan Škoda Power, Tylova 1/57, 301 57, Pilsen, Czech Republic
}

\begin{abstract}
An investigation of one and half axial turbine stage configuration was carried out in a closedloop wind tunnel. The investigation was addressed to that impact how the previous stage outlet flow field influences the flow structures in the next stator in steam multistage turbines. The stage - stator interaction has been studied in this work. The detailed measurement with a pneumatic probes and fast response pressure probes behind the rotor and the second stator were performed to gain the useful data to analyze the impact. The detailed flow field measurement was carried out in the nominal stage regime (given by the stage isentropic Mach number 0.3 and velocity ratio $u / c 0.68$ ). The clocking effect of the stators is discussed and detailed unsteady flow analysis is shown.
\end{abstract}

\section{Introduction}

This work is a continuation of a long-term cooperation in turbine aerodynamics research with the leading producer of steam turbines Doosan Škoda Power (DSPW). DSPW has recently expanded a spectrum of stages offered by a reaction stages used for high-pressure and mediumpressure steam turbines. A multistage arrangement design has to be done with the most accurate models. These models must include kinetic energy losses caused by a profile and secondary structures and also include enthalpy changes due to a mutual interaction of stages and overflow through a shroud seals.

As a support of improvement of such design processes a research of a flow filed in a multistage turbine is realized in cooperation of VZLU and DSPW. Effects of the inlet flow parameters on a flow field structures in the axial turbine stage were studied as an initiative activity within this work. Various inlet turbulence intensity and inlet end-wall boundary layer thicknesses were studied in refs. [1, 2]. The results show that the typical steam turbine reaction stage is nearly insensitive on such inlet flow parameters changes.

At present, the main effort is directed to investigating the influence of the multi stage flow structures interaction.

The stator preceded by the full stage operated under various operational conditions and with different rotor shroud geometry configurations was experimentally studied in this work. Overall performances and time averaged results are discussed in ref. [3], investigation by an in-house CFD codes is presented in refs. [4, 5]. This contribution is focused on a specific field in multistage turbines - the effect of stators clocking. Usually, the clocking is studied with the same number of vanes in both stators. In this specific case, vane numbers are different and indivisible. The configuration was studied in order to find possible specific behaviours caused by interactions of this case.

\section{Facility configuration}

The test rig is a part of the closed-loop aerodynamic wind tunnel located in VZLU turbomachinery laboratory. A level of pressure in the wind tunnel is maintained by a system of vacuum pumps and a desired air mass flow rate is then provided by means of a twelvestage radial compressor driven by a $1.3 \mathrm{MW}$ electric motor. The air temperature and humidity are controlled by inter-stage heat exchangers and a condenser dryer, respectively.

The one and half axial turbine stage arrangement is depicted in Fig. 1. It is consisted of the stator and the shrouded rotor of the first stage which was followed by the stator of the second stage (second rotor was not present). Blade rows axial gaps were kept similar to real steam turbine geometry. The facility configuration allowed measuring the flow field structures in two planes: at the inlet and at the outlet of the second vane. The facility description is introduced in refs. $[3,6]$ more precisely.

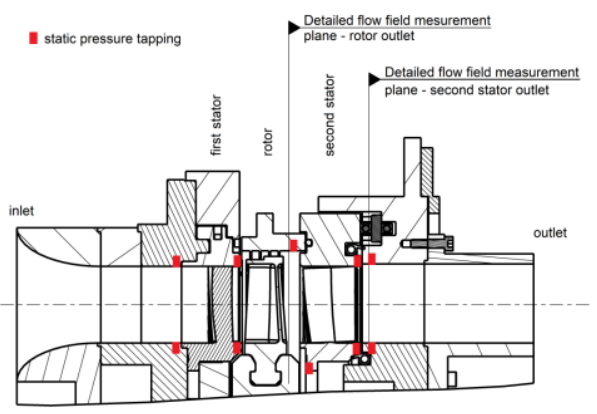

Fig. 1. The one and half stage meridian cut

\footnotetext{
Corresponding author: nemec@vzlu.cz
} 
Detailed flow field investigation was performed by a miniature five-hole pressure probes (tip diameter 1.2 $\mathrm{mm}$ ) and with a fast response pressure probes. All probes were designed and manufactured in VZLU. The design of the fast response pressure probe was focused on the measurement of unsteady total pressure. The probe combines time averaged measurements of yaw angle and static pressure with unsteady measurement of the total pressure. Dynamic calibration was performed in shock tube obtaining probe own frequency (approximately $20 \mathrm{kHz}$ ) and damping factor. Methodologies used for such fast response pressure probes development are presented in ref. [7].

\section{Turbine stage specification}

The one and half turbine stage model is consisted of the axial turbine stage REAC_v2-1 in configuration with nominal stator - rotor axial gap. The second stator geometry is REAC_v2-2. The rotor - second stator axial gap corresponds to the largest inter-stage axial gap typically used by DSPW. The blades geometry represents shaping of an experimental reaction turbine stage with increased outlet angle, which is commonly used for higher volumetric flow stages. The stage geometry is given in Tab. 1. The stage regime is specified in Tab. 2.

Table 1. Stage geometry

\begin{tabular}{|l|c|c|c|}
\hline & 1st stator & Rotor & 2nd stator \\
\hline Designation & v2-1 & v2 & v2-2 \\
\hline Vane/blade number & 100 & 92 & 56 \\
\hline Aspect ratio & 1.9 & 1.7 & 1.1 \\
\hline Pitch to chord ratio & 0.75 & 0.68 & 0.75 \\
\hline Inlet pitch angle & 90 & 70 & 90 \\
\hline
\end{tabular}

Table 2. Stage regime

\begin{tabular}{|l|c|}
\hline Stage isentropic Mach number & 0.3 \\
\hline Velocity ratio u/c & 0.68 \\
\hline The first stator isentropic Mach number & 0.23 \\
\hline The second stator isentropic Mach number & 0.24 \\
\hline The first stator Reynolds number & 215000 \\
\hline The second stator Reynolds number & 210000 \\
\hline
\end{tabular}

\section{Stators clocking}

As mentioned before, stators with the same vanes numbers are usually studied. In this case, numbers of vanes are different and moreover indivisible. Such geometry is the clocking insensitive in the overall performance point of view. It is caused by a variation of the first and the second stator vanes relative circumferential positions. It is depicted in Fig. 2 circumferential angle between the second vane and the closest first vane are shown here for 16 vanes of the second stator. Nevertheless, the stators interaction and influence of the relative position of vanes on the flow field is possible to study also in this case. As an advantage, it wasn't necessary to change relative position of the stators, because of the vanes relative position variation mentioned before. As a common divisor of vanes numbers is 4, many vanes relative positions are contained in one quarter of the wheel. The quarter is consisted of three periods and each period contains five relative vanes positions. These positions are shifted by $0.26^{\circ}$ between periods.

The facility enables to measure $82^{\circ}$ sector. So, the quarter is not fully covered (and also three periods are not captured completely), but, as it is shown further, this sector measurement gives reliable results.

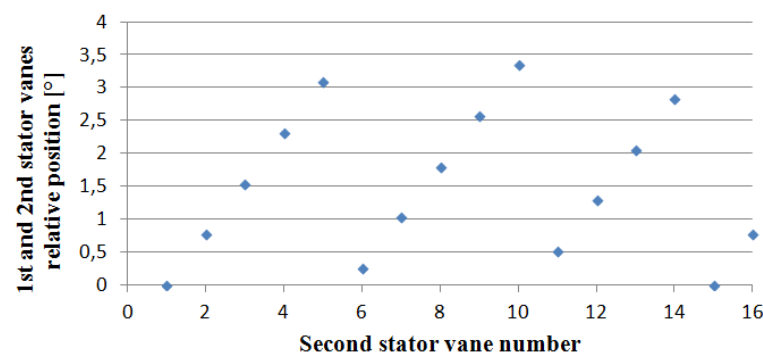

Fig. 2. The first and second stator relative vanes angles

\subsection{Time averaged characteristics}

Figure 3 shows a distribution of kinetic energy loss coefficient at the outlet of the second stator. No significant differences are possible to identify, except of vane number 4 . The same conclusion is possible to derive from radial distributions of kinetic energy loss coefficient and yaw angle (Fig. 4). Vanes numbers from 6 to 10 are drawn here. These vanes cover one period in the quarter of the stator and measurement of this sector was performed at one time, thus there is no influence of the facility regime setup. Peculiarity of the vane number 4 is also shown in the radial distributions. A damage on the vane trailing edge (on a pressure side) was found after dismounting the facility. Interesting thing is, that the kinetic energy loss coefficient radial distribution (and also integral value) was not affected a lot by the damage. On the other hand, the outlet angle was changed quite significantly. The change of the outlet angle one can derive already from the distribution of the kinetic energy loss in the Fig. 3 (the deformation of the wake in the hub region).

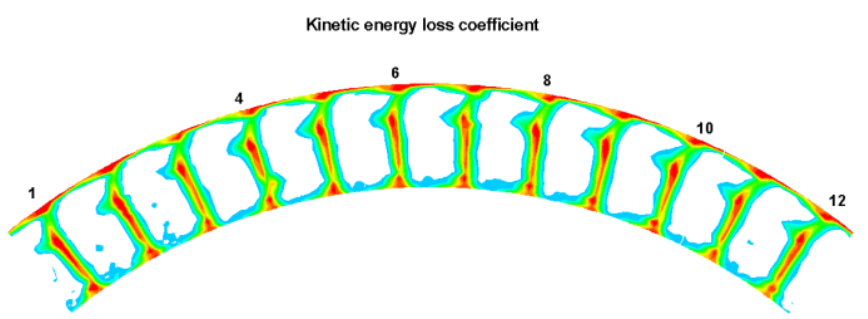

Fig. 3. The second stator kinetic energy loss coefficient distribution 
As a conclusion, the time averaged characteristics did not showed an effect of clocking on the second stator behaviours.
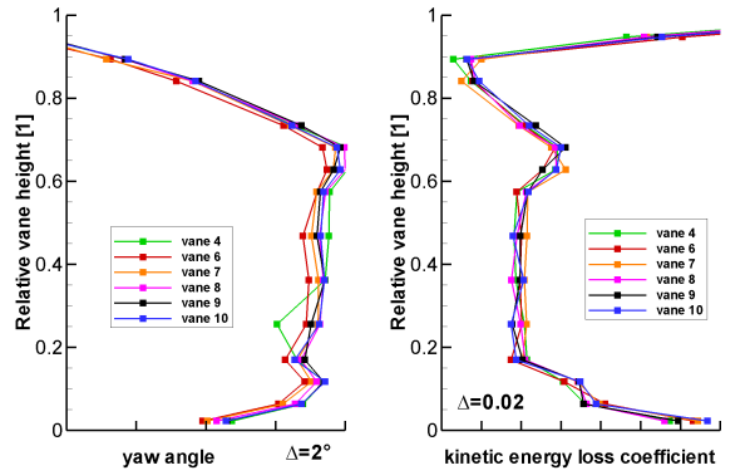

Fig. 4. The second stator outlet angle and kinetic energy loss coefficient radial distribution
(RMS) value computed from a complete signal acquired (Fig. 5). The second is RMS of a phase lock averaged signal (averaging respecting the rotor position - Fig. 6) and the third is blade passing frequency amplitude extracted with the Fast Fourier transform (FFT) (Fig. 7). Short explanation, what these results show, follows.

The simple RMS computation highlights fluctuations referenced to the second stator flow field development together with fluctuations caused by the rotor blade passing. Nevertheless, the RMS caused by the second stator flow field development is orderly higher in comparison with RMS caused by the passing of the rotor blades. So, it is possible to say, that the basic RMS shows us fluctuations mainly related to the second stator flow field development. The distribution of the basic RMS is shown in Fig. 5. Regions of a secondary flow together with wakes are clearly visible.

Total pressure RMS related to dynamic pressure

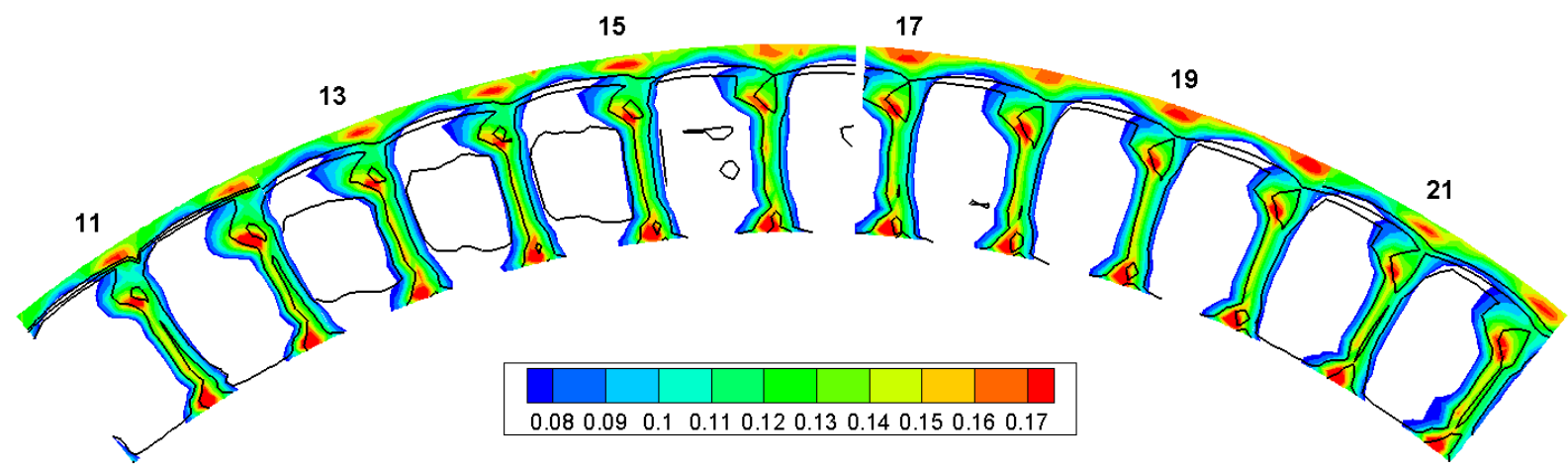

Fig. 5. RMS distribution at the second stator outlet

Total pressure RMS related to dynamic pressure - phase lock averaged

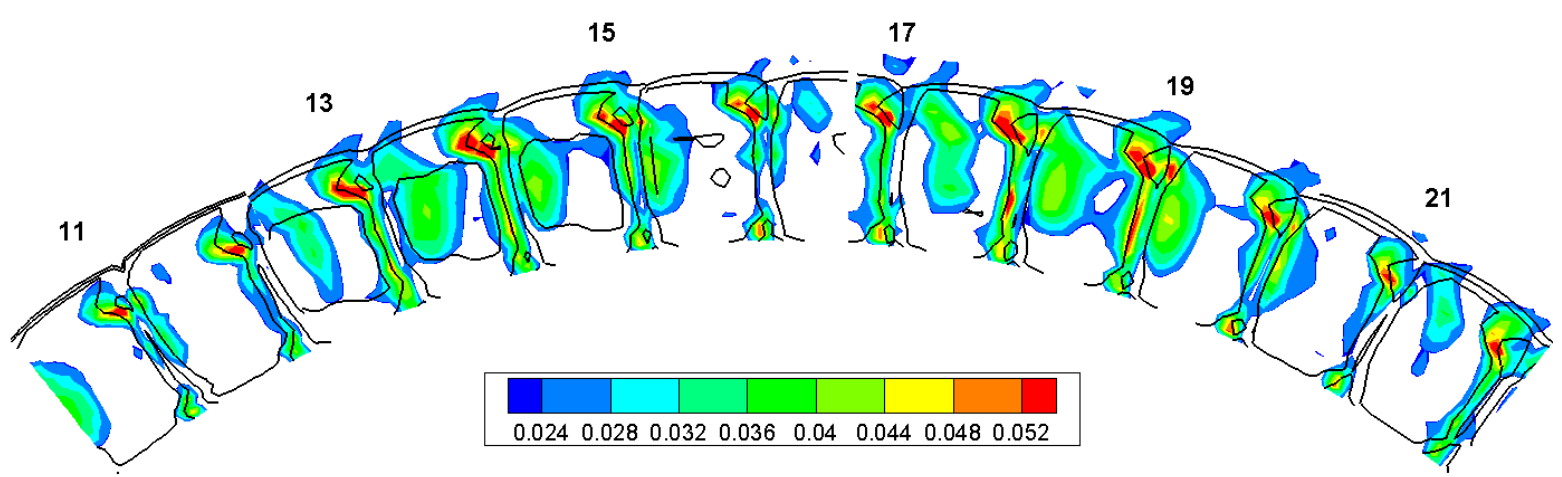

Fig. 6. Phase lock averaged RMS distribution at the second stator outlet

\subsection{Unsteady pressure measurement results}

The experiment was focused on the total pressure fluctuations capturing to analyze unsteady flow field evolution. Three main characteristics are obtained by the postprocessing. The first one is the root mean square
The RMS distribution is not distinctly affected by the stators clocking, what confirms the previous conclusion, that the second stator is not affected by the previous stator.

Phase lock averaging highlights phenomena related to the rotor flow field. An average rotor revolution is computed, then RMS value of the signal is determined. 


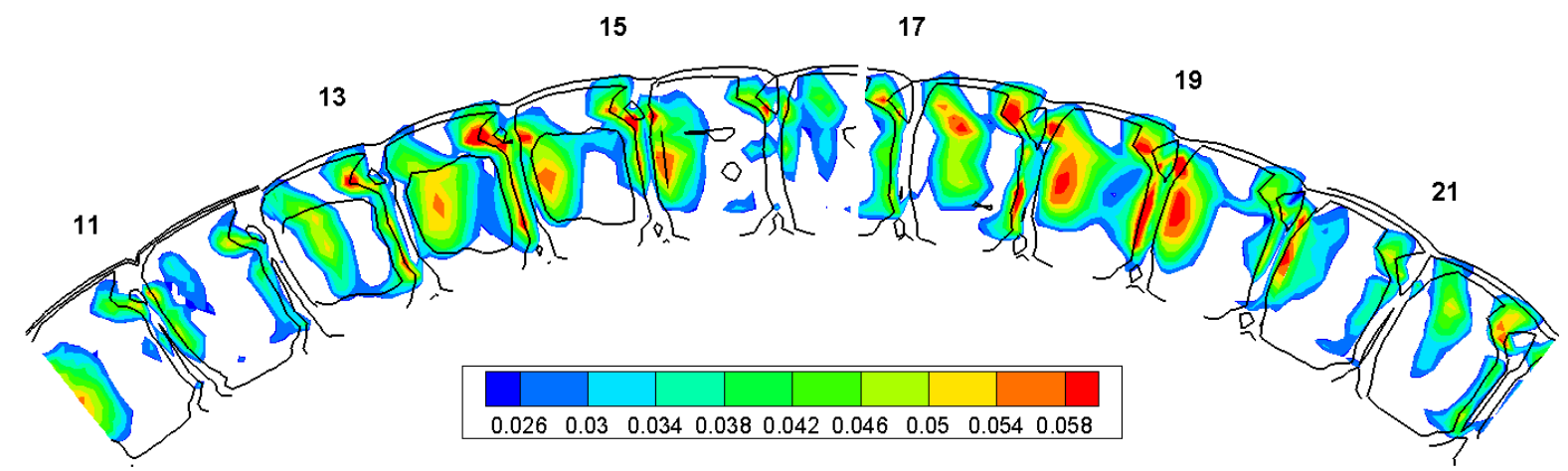

Fig. 7. Amplitude of the blade passing frequency at the second stator outlet

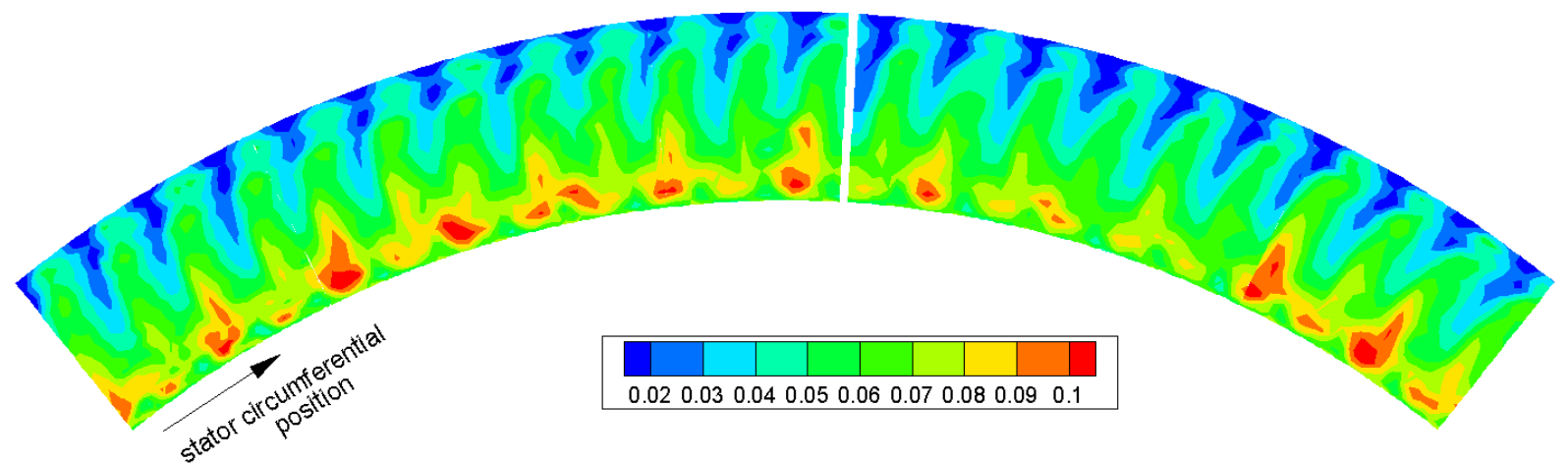

Fig. 8. Amplitude of the blade passing frequency at the rotor outlet

This RMS distribution is affected by the stators clocking, thus the clocking has an effect on the rotor flow field transformation through the second stator. Periodic changes in the flow field activity are shown in Fig. 6 and also in Fig. 7 with total pressure amplitude of blade passing frequency. The period is approximately five vanes, what is consistent with the vanes relative position variation shown in Fig. 2. With respect to the aforementioned displacement, the positions with almost identical distributions are not present exactly after five vanes, but the closest similar distributions are seen in a rhythm 554554 vanes. A zones characterised by low total pressure RMS and amplitude (vanes 11(and 12)-1621) are alternated by zones with high RMS and amplitude - vanes 14-18(and 19).

Finally, let's highlight the fact, that the most important exciting forces acting rotor behind the second stator are caused by the second stator flow field (compare RMS values in Fig. 5 and Fig. 6). Nevertheless, the unsteadiness caused by the rotor and the clocking effect on the rotor flow field transformation can bring a specific exciting frequencies due to circumferential periodic changes.

\subsection{Rotor flow field and its transformation in the second stator}

Firstly, we will focus on the flow field in the rotor outlet plane. Flow parameters distribution in this plane is given by the single stage flow field development. The rotor outlet is therefore affected only by the stator-rotor interaction and will be a reference for the transformation analysis. Figure 8 shows a distribution of total pressure amplitude (blade passing frequency) at the rotor outlet. The first stator wakes regions are clearly visible in this distribution.

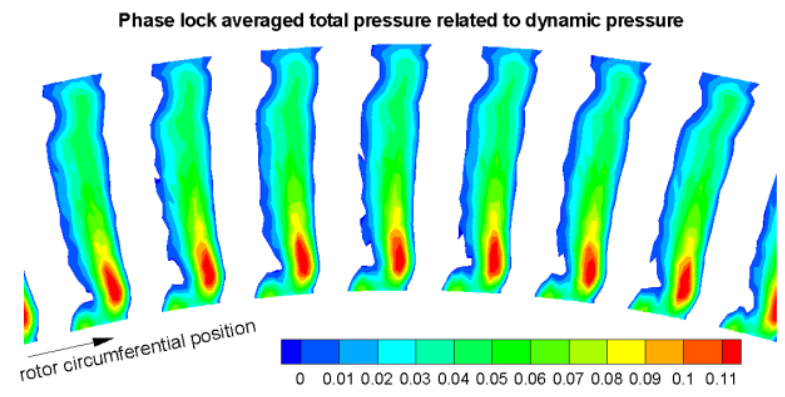

Fig. 9. Phase lock averaged total pressure difference distribution at the rotor outlet 


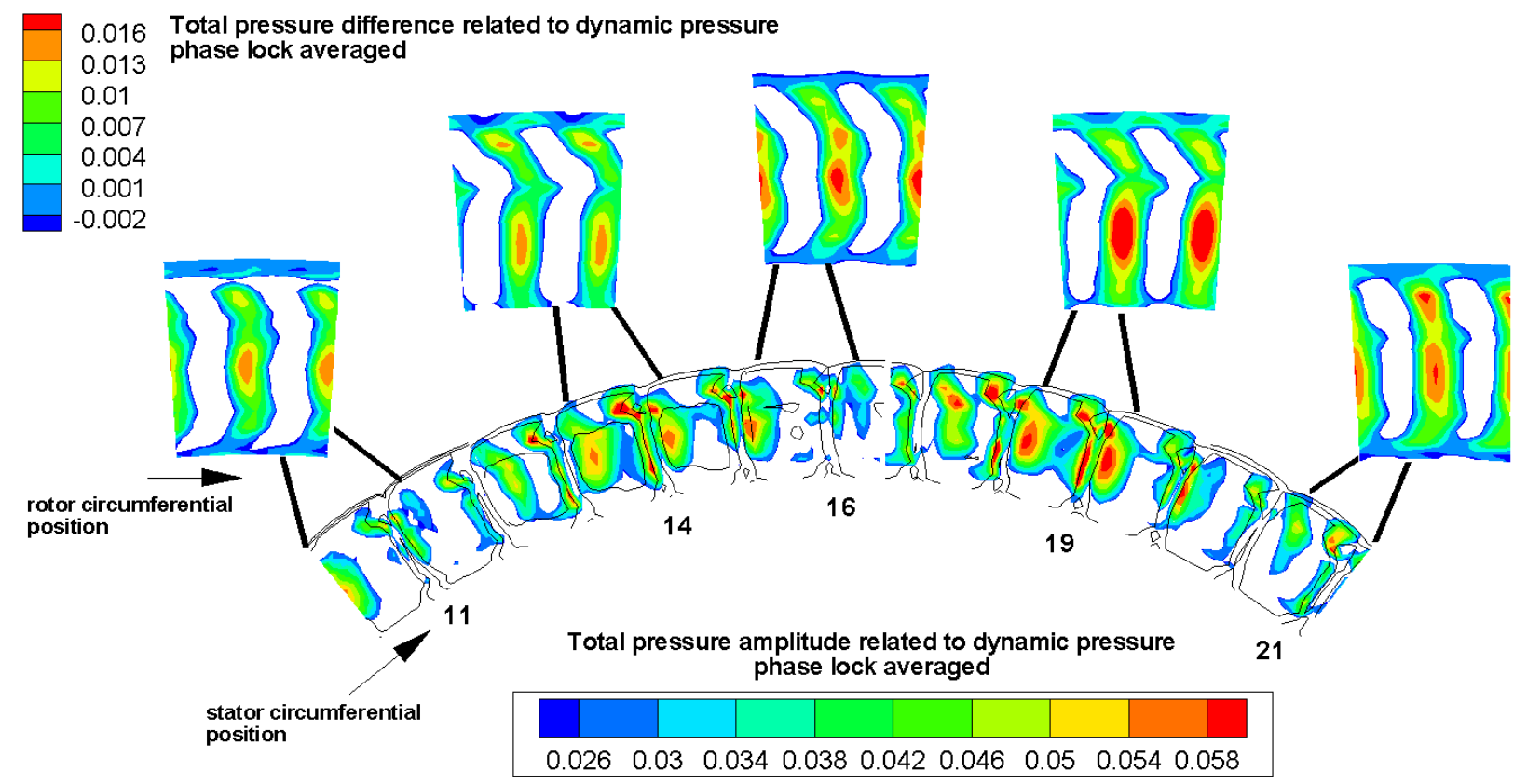

Fig. 10. Phase lock averaged total pressure difference distributions at the second stator outlet

To show the transformation process in the second stator, an averaged rotor outlet distribution is used. The averaged distribution is got by averaging across traversed area and is shown in Fig. 9. The influence of the first stator is averaged in this distribution. Let's note, that absolute values (total pressure is used here) do not provide a sufficient resolution for the rotor structures identification. Therefore, a difference between a local total pressure and total pressure averaged for a relevant diameter is used in order to highlight rotor structures. The highest total pressure amplitude is in the hub region, where the rotor hub passage vortex is developed. It is clearly visible in Fig. 9. The rotor blade wakes are clearly visible in this figure as well. One can also expect a region of high amplitude in the tip area, which should be related to the rotor tip passage vortex. In the case of the shrouded rotor, there is a mixing process with the rotor shroud leakage flow, what leads to a suppression of the tip passage vortex intensity.

Contrary to the rotor outlet plane, the second stator outlet plane is characterized by the position of the highest amplitude in the midspan and in the tip regions. Moreover, the regions are influenced also by the clocking of the stators as mentioned before. The variation of the rotor-related flow field is shown in Fig. 10. Distributions of phase lock averaged total pressure difference are shown here for a few representative areas.

\section{Conclusion}

The specific case of stators clocking was studied with unsteady pressure measurement instrumentation. It is shown, that the case with indivisible numbers of stators vanes is clocking insensitive in time averaged characteristics point of view. The clocking effect is possible to observe in unsteady evolution of the flow field. Specific frequencies of exciting forces appear due to an interaction of stators and rotor flow fields.

It is shown, that the second stator moves the unsteadiness caused by the rotor hub passage vortex in the tip direction, what may be interesting namely for a following rotor excitation. The forces caused by these unsteady behaviours are likely to be orderly lower in comparison with exciting by the second stator. Thus, wakes and secondary flow regions of the second stator stays the most important for the following rotor excitation.

\section{Acknowledgment}

The work was done with financial support of the Technology Agency of the Czech Republic on the project TA04020129 "Investigation of flows in a multistage axial turbine stages arrangement".

Authors thanks to Doosan Škoda Power for permission to publish these results.

\section{References}

1. T. Jelínek, P. Straka, V. Uruba, AIP Conf. Proc. 1745, 020019 (2016)AIP, 2016

2. T. Jelínek, M. Němec, V. Uruba, 22nd International Conference Engineering Mechanics 2016, pp. 254257, 2016

3. M. Němec, T. Jelínek, P. Milčák, 16th conference on Power System Engineering, Thermodynamics \& Fluid Flow - PSE 2017, 2017

4. P. Straka, J. Pelant, Engineering Mechanics 2016, pp. 522-525, 2016

5. P. Straka, J. Pelant, M. Němec, T. Jelínek, P. Milčák, EPJ Web of conferences, Vol. 143, 2016

6. T. Jelínek, M. Němec, Turbostroje 2010, 2010

7. M. Němec, PSE-ES 2009, 2009 